Káñina, Rev. Artes y Letras, Univ. Costa Rica XL (Extraordinario): 105-122, 2016 / ISSN:2215-2636

\title{
TEVI: TECLADO VIRTUAL COMO HERRAMIENTA DE ASISTENCIA EN LA COMUNICACIÓN Y EL APRENDIZAJE DE PERSONAS CON PROBLEMAS DEL LENGUAJE VINCULADOS A LA DISCAPACIDAD MOTRIZ
}

\author{
TEVI: A VIRTUAL KEYBOARD TO SUPPORT COMMUNICATION AND LEARNING FOR PEOPLE \\ WITH PHYSICAL AND LANGUAGE DIFFICULTIES
}

\author{
Ligia Jácome Amores*
}

Janio Jadán-Guerrero**

\begin{abstract}
RESUMEN
Este artículo presenta una investigación empírica sobre el impacto de un sistema computacional denominado TEVI (Teclado Virtual) diseñado para facilitar la comunicación de niños con problemas de lenguaje a causa de una discapacidad física. La metodología utilizada en la investigación se basó en cuatro fases: en la primera fase, se realizó una investigación exploratoria mediante encuestas y entrevistas no estructuradas a autoridades y a diez maestros de dos centros de educación especial en dos provincias del Ecuador. En la segunda fase, se desarrolló el sistema computacional. El sistema se estructuró en tres módulos: entrada de datos (Interfaz de usuario), procesamiento de información (Gestor de base de datos) y salida de datos (Algoritmos de procesamiento de lenguaje natural). En la tercera fase, se efectuó una evaluación preliminar del prototipo con especialistas y una persona con discapacidad motriz. Finalmente, en la cuarta fase se llevó a cabo una evaluación experimental en la que participaron 75 niños de edad escolar, entre ellos el $80 \%$ con problemas de lenguaje por su discapacidad física. El instrumento de evaluación contempló un panel de pictogramas y un teclado virtual con texto predictivo. Los tiempos registrados en las actividades planteadas con los maestros y luego de un análisis estadístico lineal general univariante se pudo evidenciar una disminución del $57.3 \%$ en el tiempo de ingreso de información usando el panel de pictogramas frente al teclado virtual. Un análisis cualitativo permitió recoger eventos y comentarios de maestros, quienes manifestaron que TEVI constituye una herramienta potencial para desarrollar estrategias de aprendizaje.

Palabras clave: TEVI, Teclado Virtual, comunicación, discapacidad física, accesibilidad.
\end{abstract}

\begin{abstract}
This article describes an empirical research on the impact of a computer system called TEVI (Virtual Keyboard) designed to facilitate communication for children with language problems caused by a physical disability. The methodology used in the research was based on four phases: in the first phase, an exploratory research was conducted through surveys and unstructured interviews to authorities and ten teachers from
\end{abstract}

\footnotetext{
* Universidad Tecnológica Indoamerica. Facultad de Ingeniería en Sistemas. Ecuador. Correo electrónico: ligiajacome@uti.edu.ec

** Universidad de Costa Rica. Centro de Investigaciones en Tecnologías de la Información y Comunicación. Costa Rica. Correo electrónico: janio.jadan@ucr.ac.cr Recepción: 15/1/2016. Aceptación: 16/3/2016
} 
two special education schools in two provinces of Ecuador. In the second phase, the computer system was developed. The system is divided into three modules: input (User interface), data processing (database manager) and output (Natural Language Processing algorithms). In the third phase, a preliminary evaluation of the prototype is made with specialists and a person with motor disabilities. Finally, in the fourth phase was carried out an experimental evaluation in which 75 children of school age, including the $80 \%$ with language problems because of their physical disabilities. The instrument for evaluation has a panel of pictograms and a virtual keyboard with predictive text. The time registered in the activities with teachers was processed with a general linear univariate statistical analysis. The results showed a decrease of $57.3 \%$ of the time in the input of information using the panel of pictograms compared to the virtual keyboard. In addition, a qualitative analysis allowed collecting events and comments from teachers who said that TEVI is a potential tool to develop learning strategies.

Keywords: TEVI, Virtual Keyboard, communication, physical disability, accessibility.

\section{Introducción}

La comunicación es un proceso consciente que permite transmitir información entre dos o más personas. Los seres humanos usamos el lenguaje oral como principal medio de comunicación. El lenguaje, entendido como el conjunto de recursos lingüísticos comunes a todos o la mayoría de los hablantes de un idioma (Cordero Monge, 2009: 75), siempre ha sido considerado como el elemento básico de la comunicación y la interacción social. Asimismo, el habla es una acción individual, voluntaria y de inteligencia que una persona hace uso para comunicarse mediante la articulación de palabras. Sin embargo, no todas las personas pueden utilizar esta facultad para comunicarse con los demás, como es el caso de personas con alguna condición de discapacidad.

La discapacidad es una de las causas que impiden que una persona pueda articular palabras y comunicarse con su entorno. El no poder comunicarse afecta su calidad de vida y limita su desarrollo personal, educativo o profesional. En situaciones como estas, las personas necesitan la ayuda de familiares o herramientas que les ayuden a suplir su déficit dependiendo de su condición de discapacidad.

Actualmente, el avance tecnológico ha permitido que se desarrollen herramientas que ayuden a la comunicación de estas personas con su entorno. Sin embargo, no es sencillo para algunas condiciones de discapacidad, como en el caso de discapacidad física o «diversidad funcional motriz»; que es la disminución o ausencia de las funciones motoras o físicas (Brogna, 2009: 34). Muchas veces, además de la limitación física, algunas personas presentan problemas con la articulación de palabras, lo que complica aún más su capacidad para comunicarse. En este escenario, las tecnologías de la información y comunicación (TIC) constituyen una posible alternativa de solución, siempre y cuando se adapten a las necesidades funcionales y económicas de estas personas.

En países en vías de desarrollo como Ecuador, la creación de tecnologías de código abierto o de bajo costo ayudaría a mitigar en algo este problema, especialmente en centros educativos que se ven limitados en el uso de la tecnología por razones de costo y de software especializado. En los centros de educación especial es más evidente la escasez de recursos técnicos para asistir a sus alumnos con capacidades diferentes en la comunicación y el aprendizaje en el aula.

Esta investigación se enfoca en este problema como una oportunidad para desarrollar una herramienta tecnológica TEVI (Teclado Virtual) que ayudará a niños con problemas de lenguaje por su condición de discapacidad física; 
$\mathrm{y}$, de esta manera, puedan mejorar su comunicación y aprendizaje en el aula.

El artículo está estructurado de la siguiente manera: comienza con los antecedentes de la problemática en Ecuador. Luego, se citan las principales técnicas y trabajos relacionados en esta área. Posteriormente, se explica la metodología que se utilizó en la investigación. Seguidamente, se detalla la arquitectura del Teclado Virtual TEVI y su relación con el campo de procesamiento de lenguaje natural. Finalmente, se reportan los resultados de una evaluación empírica y se presentan las conclusiones.

\section{Antecedentes}

Ecuador se ha convertido en un referente a nivel latinoamericano en políticas de inclusión social, gracias a que el gobierno ha impulsado nuevas reformas y programas en el área de la salud, vivienda, trabajo digno, acceso a servicios básicos y educación a través del Plan Nacional del Buen Vivir 2013-2017 en su objetivo 3 (Secretaría Nacional de Planificación y Desarrollo, 2013). Si bien es cierto que los indicadores del mejoramiento de la calidad de vida de las personas con discapacidad han mejorado, aún queda mucho por hacer. En el área de inclusión educativa, de especial interés en la presente investigación, aún existen barreras sociales, culturales y tecnológicas. A continuación, se resumen las posibles causas.

\subsection{El entorno familiar}

Cuando un niño o niña con capacidades diferentes nace en el núcleo de una familia, independientemente de la condición social, trae consigo incertidumbre y desesperanza. Muchas familias no saben cómo afrontar esta situación, los miembros sienten impotencia de hacer poco o nada por sus hijos con discapacidad y a veces este escenario trae consigo hogares disfuncionales o conflictivos. El problema crece y profundiza cuando existe un nivel socioeconómico bajo, que dificulta a las familias buscar mecanismos de ayuda en la rehabilitación de sus hijos con discapacidad. Sin embargo, este escenario está cambiando en Ecuador, gracias a la difusión de las políticas de gobierno. Las familias poco a poco están acudiendo a instituciones o programas sociales como Misión Manuela Espejo y Joaquín Gallegos Lara (Acurio Recalde, 2013: 26) para adquirir algún tipo de asistencia, rehabilitación o insumos que les permita mejorar su calidad de vida.

\subsection{Centros educativos}

La Ley Orgánica de Educación Intercultural en el art. 47 establece: «El Estado ecuatoriano garantizará la inclusión e integración de las personas con discapacidad eliminando las barreras de su aprendizaje», por lo que las instituciones de educación regular están obligadas a recibir a alumnos con discapacidad creando adaptaciones físicas y curriculares (Ley Orgánica de Educación Intercultural, 2011). Sin embargo, los maestros de estas instituciones no están preparados para trabajar con alumnos con diferentes capacidades al mismo tiempo, pues estos alumnos requieren de metodologías y de material didáctico y tecnológico acorde con su condición o tipo de discapacidad. Además, son pocos los alumnos que han sido incluidos en centros de educación regular y la gran mayoría siguen acudiendo a los centros de educación especial, sintiéndose todavía discriminados o marginados, por lo que la inclusión educativa no ha sido aplicada en su totalidad en el país.

\subsection{Accesibilidad tecnológica}

En Ecuador, el acceso a la tecnología ha sido un proceso un tanto demorado, especialmente en sectores urbanos marginales y rurales. Por un lado, existen niños que se han convertido en nativos digitales porque tienen contacto temprano con la tecnología (Rodríguez, 2002: 14); pero por otro lado, existen niños en hogares de bajos recursos económicos que les resulta más complicado acceder a esta, lo cual ha generado una brecha en la accesibilidad tecnológica.

Los datos publicados en la Encuesta de Tecnologías de la Información y la Comunicación del Instituto Nacional de Estadística y Censos 
(INEC, 2013), muestran que el acceso de los hogares a Internet en el país ha incrementado, al pasar del 11,8 \% en el 2010, al 28,3\% en el 2013.

En el área urbana, los hogares que tienen acceso al Internet se han incrementado del 16.7 $\%$ en el 2010, al 37,0 \% en el 2013; mientras que, en el área rural el incremento ha sido del $1,3 \%$ en el 2010, al 9,1\% en el 2013 (INEC, 2013).

Este crecimiento en la accesibilidad tecnológica en el Ecuador en los últimos años puede ser la oportunidad para que las familias que trabajan en colaboración con los maestros debidamente capacitados, puedan crear mecanismos que aporten a la educación inclusiva, como utilizar herramientas, muchas de ellas gratuitas y fácilmente adaptables al contexto y realidad, que fortalezcan la comunicación y el aprendizaje de los niños con capacidades diferentes.

Niños con problemas del lenguaje a causa de la discapacidad motriz.

Según el Consejo Nacional para la Igualdad de Discapacidades (CONADIS, 2013); el Ecuador tiene el $2.5 \%$ de su población con una condición de discapacidad; y de este grupo poblacional la discapacidad más frecuente es la física, con un 47,39\%. La discapacidad del lenguaje como tal tiene apenas el 1,36\%. Sin embargo, no se registran porcentajes en los cuales los problemas del lenguaje se presenten por consecuencia de otras condiciones físicas o intelectuales, que puedan generar problemas en la articulación de las palabras (CONADIS, 2013).

En el cuadro 1, se muestra un resumen de las estadísticas del tipo de discapacidad en el Ecuador.

\section{CUADRO 1}

Distribución Porcentual de Discapacidad en Ecuador

\begin{tabular}{lcc}
\hline Tipo de discapacidad & Población & Porcentaje \\
\hline Lenguaje & 5,620 & $1,36 \%$ \\
Psicológica & 18,125 & $4,38 \%$ \\
Visual & 48,836 & $11,79 \%$ \\
Auditiva & 52,780 & $12,74 \%$ \\
Intelectual & 92,567 & $22,35 \%$ \\
Física & 196,312 & $47,39 \%$ \\
\hline Total & 414,240 & $100 \%$ \\
\hline
\end{tabular}

De la población con discapacidad motriz, el $2.4 \%$ corresponde a la provincia del Tungurahua y el $0.97 \%$ a la Provincia de Napo, que son las dos provincias que aborda la presente investigación, cuyo objetivo principal es apoyar la inserción tecnológica a niños con discapacidad motriz y problemas de lenguaje.

Para llevar a cabo la investigación empírica se planteó como propuesta el desarrollo de un software de código abierto denominado TEVI (Teclado Virtual). En el prototipo se consideraron algoritmos relacionados con el procesamiento del leguaje natural (PLN, por sus siglas en inglés), tales como la generación de texto predictivo y la sintetización de voz a través de librerías Text-to-Speech. En la fase experimental participaron en total 75 niños con dificultades del lenguaje como consecuencia de sus problemas de movilidad. Los niños pertenecen a dos centros de educación especial de las dos provincias anteriormente señaladas.

\section{Trabajos relacionados}

La comunicación es un proceso fundamental para fortalecer la autonomía de las personas con capacidades diferentes. El desarrollo psicomotor en las personas, desde edades tempranas, favorece a que de adultos se valgan por sí mismas en sus actividades diarias. El término 'desarrollo psicomotor' hace referencia a la adquisición de una serie de destrezas que un niño fortalece en diferentes áreas, tales como, la motricidad general, lenguaje y adaptación social (Robles Bello y Sánchez Teruel, 2014), en los tres primeros años de edad y de forma progresiva. Si un niño por su condición motora limitada no puede articular palabras, no significa que no pueda comunicarse; ya que el avance tecnológico ha permitido el desarrollo de otros mecanismos para suplir el déficit en la comunicación.

Entre las soluciones que se han encontrado están los Sistemas Aumentativos y Alternativos de Comunicación (SAAC), que son herramientas tecnológicas de asistencia para personas que no pueden emplear la lingüística oral y que en su lugar usan canales de comunicación en los cuales se aplican técnicas de la lingüística no 
verbal (signos, símbolos, pictogramas) y/o canales no lingüísticos (gestos, expresión facial, mirada, actitud postural). Estas herramientas poseen interfaces que incluyen comandos de voz o pulsadores de tamaño y ergonomía adaptados, que facilitan la transmisión de una idea o un mensaje para su interacción con el entorno (Prabhu, 2009).

Los SAAC aplican complejos sistemas de sensores y electrodos (Barreto et al., 2000: 53), que interpretan señales $E M G$ o electromiografías de algunos músculos faciales o bucales (Kumaran et al., 2007) para controlar el puntero del ratón mediante la detección de movimientos simples. Sistemas más sofisticados usan señales $E E G$ o electroencefalografías, para detectar la actividad cerebral e identificar una figura, un color o una posición del cursor en la pantalla (Pinheiro et al., 2011). Estos sistemas usan una interfaz cerebro-computador (BCI, por sus siglas en inglés) (Wolpaw et al., 2002; Klever y Birbaumer 2005: 65). Existen también sistemas que interpretan señales $E O G$ o electrooculografías, que son aquellas que controlan el puntero del ratón por medio de la dirección del movimiento del ojo (González et al., 2013: 313). Estos últimos se basan en la técnica 'eye tracking', la cual detecta cambios en la mirada y/o parpadeo (Kro'lak y Strumiłło, 2015: 335; Bradski et al., 2005: 119). Adicionalmente, se pueden encontrar sistemas basados en las características de Haar (Haar-like features en inglés), que son algoritmos de seguimiento de características faciales, como el color de la piel, tamaño de ojos, cejas, boca o nariz (Kro'lak y Strumiłło, 2009: 133).

En la interacción tradicional con un computador, además de los movimientos del ratón, se usa el teclado para el ingreso de información. En cambio, en los sistemas alternativos de comunicación se suelen utilizar teclados virtuales, los cuales consisten en una interfaz gráfica que simula un teclado QWERTY en pantalla (Perrinet et al., 2009: 38; Zhai et al., 2000). Con el aparecimiento de los dispositivos móviles los teclados virtuales también se han popularizado y cada vez se desarrollan novedosos algoritmos de predicción de texto, reconocimiento de voz o movimiento de los ojos para agilitar el proceso de ingreso de información.
Estos avances tecnológicos en conjunto con las tecnologías de procesamiento de señales EEG y EMG constituyen un reto importante para desarrollar herramientas de mayor alcance y utilidad, en beneficio de personas que no pueden comunicarse mediante el habla debido a su discapacidad motriz (Pinheiro et al., 2011).

En el ámbito educativo, los sistemas alternativos de comunicación incorporan elementos adicionales que apoyan la interacción y motivación para fortalecer el aprendizaje de los niños. En este contexto, se aplican sistemas de reconocimiento automático de voz (ASR: Automatic Speech Recognition) y la generación de voz por computadora (TTS: Text-to-Speech Synthesis). En relación con los primeros, estos convierten la voz humana en texto usando técnicas de modelado acústico y decodificación (Agüero, 2012). En cambio, los segundos procesan una cadena de texto y la traducen a una pista de audio que suena tal y como una persona lo diría (Beck, 2004). El uso de estos sistemas ha permitido el desarrollo de interfaces amigables y accesibles, las cuales tienen la capacidad de brindar asistencia y retroalimentación a través de mensajes de voz, con el fin de que haya interés por parte del niño y desarrolle interacción comunicativa (Robles Bello y Sánchez Teruel, 2014). A continuación se presentan algunas iniciativas en este campo.

DIGALO (Herramienta de apoyo básico para estudiantes de L2). Es una herramienta computacional destinada a apoyar el aprendizaje del léxico y la escritura de estudiantes principiantes de L2 (Leoni de León, 2011).

HANEN (Hablando nos Entendemos los Dos). Desarrollado en Toronto (Canadá) para niños de hasta cinco años que se comunican de manera no verbal, y para aquellos que están comenzando a combinar dos o tres palabras (Maggio, 2004).

PLAPHOONS. Creado por Jordi Lagares, es un programa de comunicación dinámico que funciona usando una serie de dibujos que representan acciones deseos o sentimientos para construir mensajes (Robles Bello y Sánchez Teruel, 2014).

PEAPO (Programa de Estructuración Ambiental Por Ordenador). Es una base de datos de 60 pictogramas que representan actividades 
o entornos de la vida escolar y familiar, el cual permite tener un grado de autonomía (Pérez de la Maza, 2002).

Sc@UT. Es un sistema aumentativo que mejora la autonomía personal de fácil aprendizaje e interfaz intuitiva y que facilita la labor de profesores y padres de familia (Espejo Cárdenas et al., 2009).

ABLAH. Creado en el 2010, como un sistema de comunicación aumentativa móvil, cuya función es simplificar el sistema de comunicación y aprendizaje para personas con autismo (Robles Bello y Sánchez Teruel, 2014).

Sin lugar a duda, las tecnologías y herramientas que se han hecho referencia en este apartado contribuyen de manera positiva en aspectos de comunicación, rehabilitación y aprendizaje. No obstante, poco se conoce de la aplicación específica en el ámbito educativo con niños quienes tienen problemas de comunicación, debido a su discapacidad motriz. Este hecho dio sustento para llevar a cabo una investigación preliminar en Ecuador, que permita conocer de cerca los problemas y necesidades en este ámbito.

\section{Investigación preliminar}

Para la recopilación de la información sobre los problemas del lenguaje en la discapacidad física, el grupo de investigadores realizó una indagación preliminar en la zona 3 de Ecuador, para conocer que los Centros de Educación Especial Ambato y Maximiliano Spiller ubicados en las Provincias del Tungurahua y Napo respectivamente son los únicos centros que tienen un mayor porcentaje de alumnos con discapacidad física-cognitiva, y la gran mayoría de estos niños con problemas de comunicación hablada.

Se realizaron entrevistas con los directores de estos centros y como resultado se constató que la discapacidad más frecuente era la motrizcognitiva. Además, se mostró que el $80 \%$ de los niños con discapacidad motriz presentaban problemas en la comunicación, lo cual limita el proceso de aprendizaje en el aula. Adicionalmente, indican que la falta de recursos y material didáctico es el mayor inconveniente en la rehabilitación y el desarrollo de destrezas cognitivas. También, estos directores manifestaron que la inserción de recursos tecnológicos, didácticos y terapéuticos podría favorecer en gran medida el aprendizaje de los alumnos y la labor de los maestros. Sin embargo, en sus centros educativos los recursos que se disponen resultan insuficientes para llevar a cabo tal misión.

Posteriormente, se encuestó a diez maestros de educación especial de los dos centros focalizados. El objetivo era determinar la metodología de enseñanza utilizada en el aula, así como experiencias con los alumnos con discapacidad motriz y problemas de lenguaje. Por un lado, los maestros concuerdan con que educar a niños con esta condición es una tarea complicada, ya que presentan problemas de comunicación y se requiere que la formación sea más personalizada. Por otro lado, indican que les resulta complejo trabajar con escasos recursos tecnológicos y que el material utilizado en el aula es básico e insuficiente, lo cual limita desarrollar las destrezas cognitivas y comunicativas en los niños. Las encuestas también reportan un alto grado de aceptación de una propuesta tecnológica que apoye a su labor en el aula. En las preguntas abiertas manifestaron que una herramienta tecnológica ayudaría a fortalecer la rehabilitación del lenguaje. Consecuentemente, serviría para que los niños puedan comunicarse más rápidamente y fomentaría la interacción en el aula, tanto con sus compañeros como con sus maestros.

Finalmente, se hicieron conversatorios no estructurados con padres de familia y terapeutas de los centros educativos. Al conocer sobre la idea de usar una herramienta tecnológica con sus hijos mostraron entusiasmo. Además, manifestaron que la herramienta podría incorporarse en sus hogares y, además, ayudaría a fortalecer el trabajo realizado por los terapeutas y maestros en la escuela.

Con la información recopilada de los directores, maestros, terapeutas y padres de familia se evidenció la necesidad de proponer una solución tecnológica adaptada a la realidad ecuatoriana, en favor de los niños con 
dificultades del lenguaje debido a su discapacidad física.

\section{TEVI (Teclado Virtual)}

Esta propuesta tecnológica consiste en un teclado virtual denominado TEVI, el cual facilita la comunicación y aprendizaje de niños con discapacidad física y dificultades del lenguaje.

En este apartado se presenta la arquitectura de TEVI, los aspectos de la interfaz y la interacción con el usuario, y los primeros resultados de una evaluación preliminar del prototipo.

\subsection{Arquitectura de TEVI}

TEVI consta de tres módulos: en primer lugar, el módulo de entrada que está compuesto de un teclado virtual, un panel de pictogramas y una cámara web. En segundo lugar, el módulo de procesamiento de información compuesto por el Gestor de Datos y la Base de Datos en sí. Finalmente, el módulo de salida compuesto por el componente de Procesamiento de Lenguaje Natural (PLN), el Generador de Lenguaje Natural (GLN) y el sintetizador de voz.

La figura 1 muestra el diagrama de diseño de TEVI, sus módulos y el flujo de información.

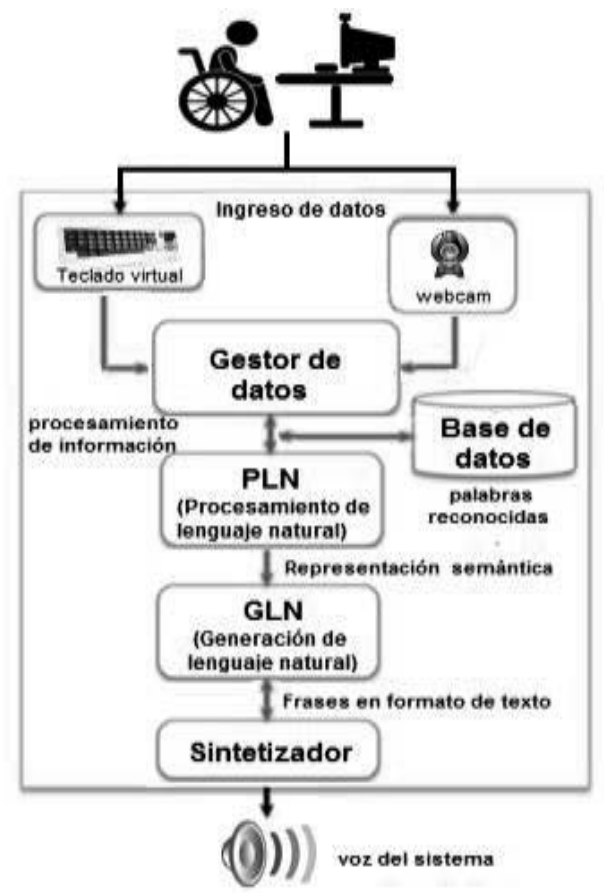

FIGURA 1

Diagrama de diseño de TEVI (Teclado Virtual).

En el desarrollo del software se utilizó la metodología de programación extrema o eXtreme Programming (XP) propuesta por Kent Beck (Kent, 2004). Esta metodología permitió adaptar fácilmente los requerimientos de la interfaz de usuario y componentes de ingreso de información del primer módulo. Para el almacenamiento y procesamiento de información se utilizó el motor de base datos SQLite (SQLite Copyright, 2014), el cual se caracteriza por ser liviano y por almacenar la información directamente en archivos de disco. Esto favorece a la portabilidad del software, tanto a nivel de plataforma (Windows, Linux, Mac) así 
como la portabilidad entre arquitecturas de 32 y 64 bits. Finalmente, para el componente de Procesamiento de Lenguaje Natural se utilizó la librería System.speech.dll de Microsoft .Net. Esta librería hace uso de un algoritmo TTS (TextTo-Speech), el cual recibe una cadena de texto y lo procesa junto con algunas variables adicionales, como el idioma y la fonética para generar una voz sintetizada.

\subsection{Interfaz de usuario}

La interfaz de TEVI tiene dos componentes, uno que se adapta a niños que aún no saben leer ni escribir y el ingreso de la información se hace a través de un panel de pictogramas. El otro es un teclado QWERTY virtual con funciones de texto predictivo. En ambos casos el ingreso de información es apoyado por una cámara web que captura los movimientos de la cabeza del niño para controlar el apuntador del ratón. A continuación se detallan las características de los dos compontes.

\subsubsection{Panel de pictogramas}

En el diseño del panel de pictogramas se consideraron tres aspectos importantes: accesibilidad, comunicación y aprendizaje. La interfaz provee un sistema de comunicación alternativa y aumentativa (AAC, por sus siglas en inglés). Esta característica ayuda a los niños con problemas más severos de motricidad y lenguaje puedan comunicarse más fácilmente con otras personas. En el aspecto de educación, facilita el ingreso de información a niños que están iniciando su etapa escolar y que todavía no pueden leer. La interfaz del panel muestra emocards simples y fáciles de interpretar (Desmet et al., 2012: 11), es decir, imágenes o pictogramas familiares al niño. Cada uno de ellos describe palabras o frases acerca del estado de ánimo, deseo o una acción a realizar, que son de utilidad para una comunicación más ágil; por ejemplo: 'tengo hambre', 'quiero bañarme', 'deseo descansar', etc. Es importante recalcar que para diseñar la interfaz de este componente, se hizo un estudio del contexto familiar, social y cultural de la zona. Esto permitió que se desarrollaran analogías y metáforas con elementos de su entorno. Además, junto con los expertos de los centros de educación especial se seleccionaron imágenes de acuerdo con la edad, y nivel de escolaridad. En la figura 2 se muestra el panel de pictogramas.

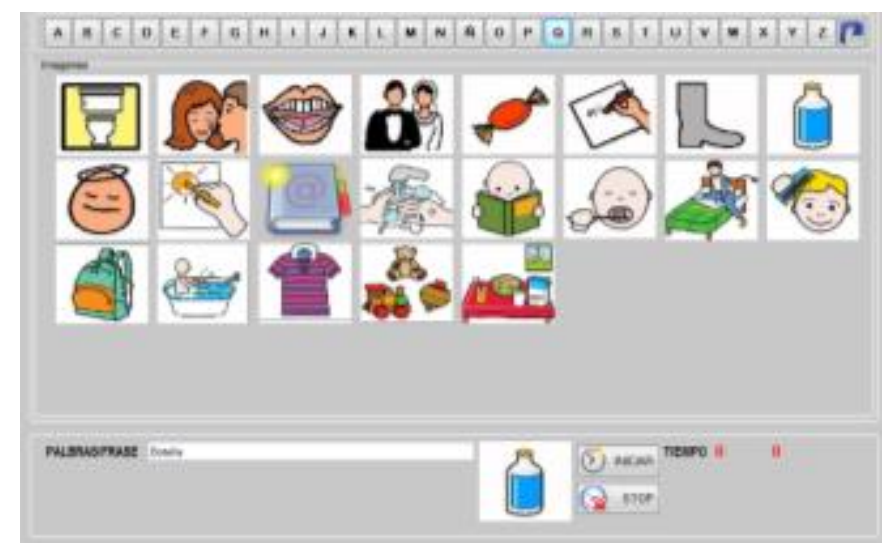

FIGURA 2

Panel de pictogramas de TEVI. 
La paleta de pictogramas puede ser personalizada según los requerimientos de un usuario, ya que cuenta con utilitarios para agregar, modificar o eliminar frases, palabras o pictogramas de la base de datos. Además, en la parte inferior de la paleta de pictogramas se ha incluido un campo de texto en el que van apareciendo las palabras o frases. Esta característica se diseñó con el fin de que el niño que aún no sabe leer vaya relacionando el pictograma con el texto. De esta manera se puede fortalecer su aprendizaje y posterior uso del teclado virtual.

\subsubsection{Teclado Virtual con texto predictivo}

El teclado virtual es similar a un teclado físico convencional QWERTY. La diferencia es que se muestra en pantalla y puede ser controlado por el mismo teclado físico del computador o por el apuntador del ratón. Generalmente, los teclados virtuales de los sistemas operativos de computador no proveen la funcionalidad de texto predictivo, esto es lo que le hace a TEVI diferente, la incorporación de texto predictivo basado en el diseño de los teclados virtuales que usan los dispositivos móviles para agilitar el ingreso de texto. El objetivo es ayudar a los niños que presentan dificultades del lenguaje, debido a su discapacidad física, a comunicarse más rápidamente.

El texto predictivo de la interfaz anticipa la palabra o frase minimizando la cantidad de teclas presionadas para formarla. La predicción se da cuando el niño empieza a ingresar las primeras letras de lo que quiere expresar. En la figura 3 se muestra el teclado virtual.

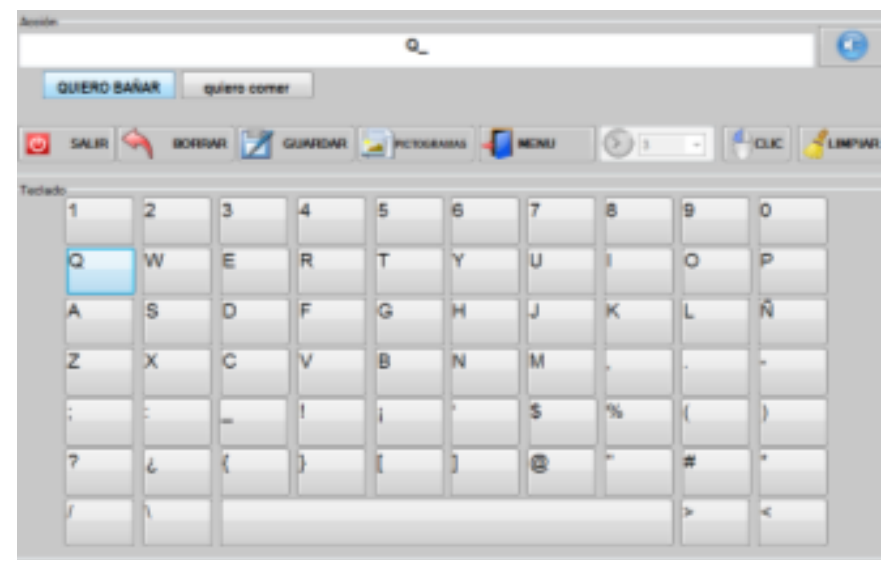

FIGURA 3

Teclado virtual TEVI con texto predictivo.

El texto predictivo de TEVI funciona haciendo referencia a una base de datos, en la cual se almacenan las palabras o frases guardadas previamente. Cuando el niño empieza a presionar las primeras letras de lo que quiere expresar, el algoritmo de texto predictivo hace una minería de palabras o frases posibles en la base de datos, de acuerdo con la combinación de teclas presionadas, y muestra una lista de opciones más probables; de las cuales el niño puede elegir de acuerdo con lo que desea comunicar. Esto resulta de gran utilidad, ya que el maestro puede ir 'entrenando' el teclado virtual cada vez que lo utilice, de tal forma que este recuerde palabras o frases utilizadas frecuentemente, haciendo que la comunicación del niño sea más rápida y eficaz. En el cuadro 2 se muestra un ejemplo. 
CUADRO 2

Generación del texto predictivo para la palabra 'bañarme'

\begin{tabular}{llll}
\hline No. Tecla & Subcadena & $\begin{array}{l}\text { Palabras desplega- } \\
\text { das }\end{array}$ \\
\hline
\end{tabular}

\begin{tabular}{llll}
1 & $|\mathrm{~b}|$ & $|\mathrm{b}|$ & bueno, bonito, ... \\
2 & $|\mathrm{a}|$ & $|\mathrm{ba}|$ & banana, baño... \\
3 & $|\tilde{\mathrm{n}}|$ & $|\mathrm{bañ}|$ & baño, bañarme... \\
4 & $|\mathrm{a}|$ & $|\mathrm{baña}|$ & bañarme \\
\hline
\end{tabular}

Como se observa en el cuadro 2 para la palabra 'bañarme', el sistema forma subcadenas con las letras de la secuencia de teclas ingresadas, y en función de cada una se filtran palabras que son desplegadas en pantalla. El algoritmo de texto predictivo para el ejemplo antes mencionado se puede resumir en 8 pasos:

1. Digita la primera letra o subcadena $|\mathrm{b}|$ en el control de edición.

2. Lee la subcadena ingresada en el control de edición y establece conexión con la Base-Datos (BD).

3. Busca en la BD todas las coincidencias (palabras o frases que empiecen con 'b') y las almacena en un arreglo.

4. Selecciona de ese arreglo de palabras y frases autocompletadas las seis opciones más probables y las presenta en la interfaz de usuario.

5. Si la palabra o frase deseada está entre estas 6 opciones, el niño selecciona y finaliza el proceso.

6. Caso contrario el niño debe digitar la siguiente letra $|\mathrm{a}|$ y regresa al paso 2 .

7. El proceso continua hasta que en la interfaz de usuario aparezca la palabra deseada: 'bañarme'.

8. Si no aparece en las $\mathrm{N}$ interacciones $(\mathrm{N}=7$ para 'bañarme'), significa que es una palabra nueva. En este caso el niño tiene la opción de guardarla para uso posterior.

El texto predictivo trabaja junto con el sintetizador de voz, ya que cada palabra o frase que el niño ingrese es acompañada por un audio que reproduce la pronunciación del texto. Esta es otra característica de TEVI que no proveen los teclados virtuales de los sistemas operativos. Con esta funcionalidad se aporta al fortalecimiento de las habilidades de lenguaje y lectoescritura del niño.

\subsection{Procesamiento de Leguaje Natural}

El Procesamiento de Lenguaje Natural juega un papel muy importante en la interacción del niño con el computador (Vallez y Pedraza, 2007), ya que incorpora elementos de reconocimiento o producción de voz haciendo que los sistemas tradicionales aumenten su capacidad (Cortez et al., 2009).

El módulo de procesamiento de lenguaje natural de TEVI utiliza la el sistema TTS (TextTo-Speech, por sus siglas en inglés) (Asterisk, 2009), específicamente la librería System.speech. dll de Microsoft .Net. El Algoritmo que se sigue es el siguiente:

1. El sistema toma como entrada la palabra, frase o expresión textual asociada al pictograma seleccionado por el niño.

2. Se realiza un análisis morfosintáctico, es decir se limpia el texto de etiquetas o caracteres especiales.

3. Se analiza cada unidad morfológica de la entrada y se determina si la estructura de la palabra o frase es correcta.

4. El sistema asigna una transcripción fonética y prosódica a cada unidad básica o palabra dentro de la frase analizada en el paso anterior.

5. El sintetizador toma las unidades fonéticas y ejecuta el procesamiento de señal digital. Este procesamiento hace una selección de unidades acústicas o de voces grabadas en la base de datos para cada unidad fonética, las cuales son concatenadas para generar de manera automática los contornos melódicos y los patrones de ritmo de las locuciones; para finalmente generar la pista de audio de salida.

En la figura 4 se esquematiza el algoritmo de TEVI como sistema TTS. 


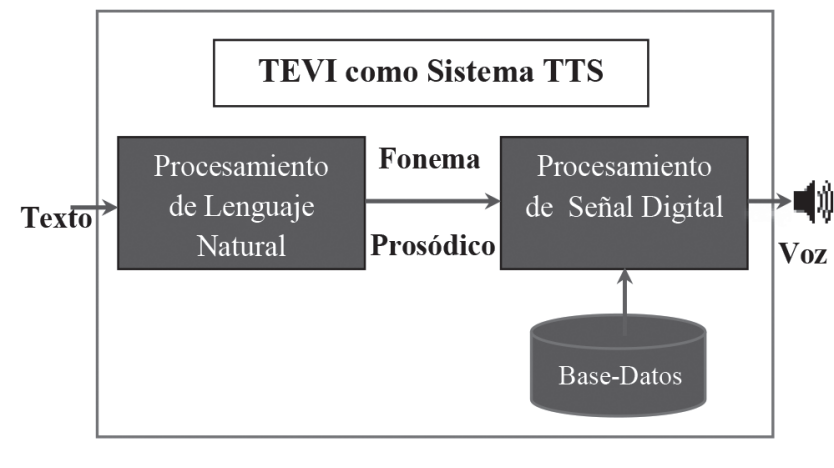

FIGURA 4

El Sistema TTS de TEVI.

En el contexto de desarrollo de TEVI, el equipo de programación realizó todas las pruebas de integración de los diferentes módulos y la evaluación del funcionamiento correcto de todos los componentes. Sin embargo, era necesario evaluar el sistema en un escenario real y para esto se diseñó una fase experimental que se describe a continuación.

\section{Fase experimental}

Con el fin de evaluar el primer prototipo de TEVI, se realizó una prueba piloto con los integrantes del equipo de investigación, especialistas y una persona adulta con motricidad limitada. Esta evaluación estaba compuesta de dos partes. En la primera parte, el participante con dificultad motriz usó el teclado virtual para ingresar palabras y frases dadas; se tomó el tiempo que tardó en cada caso mediante el cronómetro que provee el software. En la segunda parte, el mismo participante usó el panel de pictogramas de TEVI para expresar las mismas entradas (palabras y frases) pero señalando el pictograma correspondiente $\mathrm{y}$ también se registró el tiempo. Al comparar los dos tiempos se pudo evidenciar que el participante comunica sus ideas más rápidamente con el panel de pictogramas de TEVI que con el teclado virtual.

Este pilotaje inicial fue realizado para verificar que TEVI trabaja correctamente, además sirvió para mejorar la interfaz de usuario según las observaciones de los especialistas y maestros que colaboraron con esta prueba preliminar. Posteriormente, se realizó una fase experimental en un escenario real y con una muestra representativa. A continuación, se detallan los participantes y el procedimiento.

\subsection{Participantes}

En el estudio participaron tanto niños como niñas con dificultades de comunicación de dos Centros de Educación Especial (CEE), ubicados en las provincias de Tungurahua y Napo. En la Escuela Especial Ambato, se seleccionaron en conjunto con las autoridades y maestros a 35 participantes, mientras que en la Escuela Especial Maximiliano Spiller, se seleccionaron a 40 participantes. Para todos estos se elaboró un documento para solicitar el consentimiento de los padres de familia. Con la autorización de los directores de los CEE en conjunto con los maestros se recabaron todos los consentimientos informados y datos demográficos de los participantes. En el cuadro 3 se resumen tal información. 
CUADRO 3

Datos demográficos de los participantes

\begin{tabular}{lllll}
\hline CEE & Niñas & Niños & $\begin{array}{l}\text { Edad } \\
\text { Cronologica } \\
\text { M(SD) }\end{array}$ & $\begin{array}{l}\text { Nivel } \\
\text { escolaridad }\end{array}$ \\
\hline E.Ambato & 24 & 11 & $8,20(0,74)$ & $3^{\text {ro }}$ básica \\
M.Spiller & 18 & 22 & $7,18(0,40)$ & $3^{\text {ro } b a ́ s i c a ~}$ \\
Total & $\mathbf{4 2}$ & $\mathbf{3 3}$ & $\mathbf{7 , 5 8}(\mathbf{0 , 8 0})$ & \\
\hline
\end{tabular}

Es importante aclarar que no todos los participantes tenían una condición de discapacidad física con problemas de comunicación. Del total de participantes, el $4 \%$ tenían únicamente discapacidad motriz, y el $16 \%$ únicamente dificultades de lenguaje. A pesar de estos porcentajes, el experimento se llevó a cabo con el mismo procedimiento para los 75 participantes.

\subsection{Instrumento de experimentación}

Como instrumento de experimentación se usó TEVI, el cual fue instalado en quince computadoras de escritorio en la Escuela Ambato y veinte computadoras en la Escuela Maximiliano Spiller. Además, se contó con tres computadoras portátiles proporcionadas por el equipo de investigación.

En los casos de los niños con movilidad limitada severa, se utilizaron las computadoras portátiles que proveen cámara web. Para el resto de niños se usó el ratón convencional para ingreso de la información. Las tareas realizadas se describen en el siguiente apartado.

\subsection{Procedimiento}

La primera actividad llevada a cabo fue la capacitación de cuatro maestras de los dos Centros. Posteriormente, el equipo de investigación, en conjunto con las maestras, realizó varias pruebas de funcionamiento y acercamiento de TEVI a los niños. En la figura 5 se muestra a Diego, un niño de siete años con problemas del leguaje, usando el panel de pictogramas de TEVI.

Una vez que los participantes conocían el funcionamiento del instrumento de evaluación,

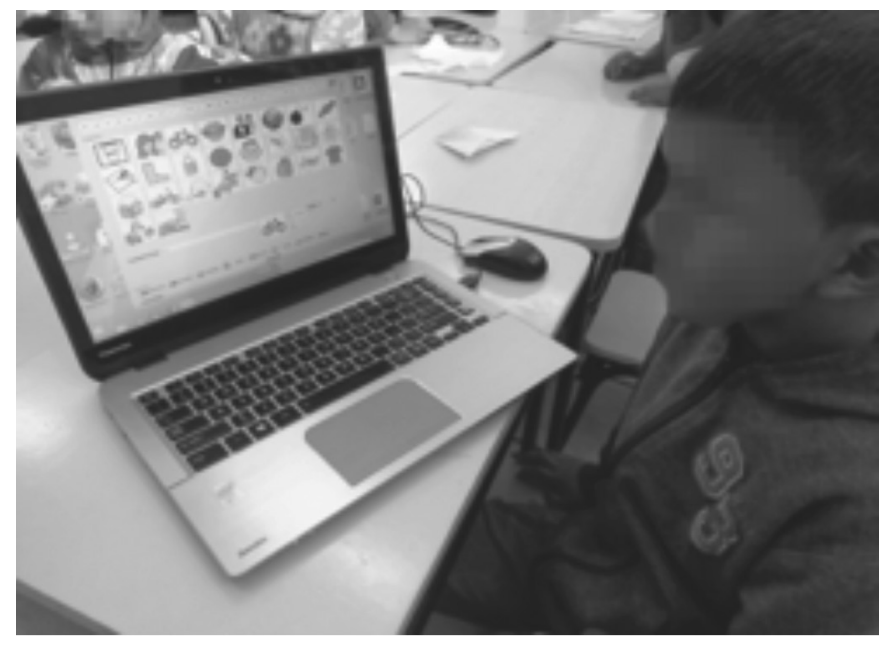

FIGURA 5

Niño con problemas del lenguaje usando el panel de pictogramas de TEVI. 
se llevó a cabo una tarea que permitió evaluar el efecto del uso de las herramientas (teclado virtual - panel de pictogramas) de TEVI. Esta tarea consistió en que cada participante, ingresara las palabras y/o frases que su maestra le indicara. En cada iteración se registró el tiempo por medio de un cronómetro que provee TEVI. Además, los observadores del equipo de investigación registraban los eventos durante la evaluación. En la segunda tarea, el participante debía ingresar la información utilizando el panel de pictogramas. La selección del pictograma hacía referencia a la misma palabra o frase que en la actividad anterior. De igual manera, el tiempo era registrado por el cronómetro de la herramienta y del equipo de investigación.

A simple vista, el equipo de investigación evidenció que, aunque las dos herramientas del sistema agilitaban la comunicación de los participantes y mejoraban la interacción en el aula, el niño tardaba menos tiempo en transmitir sus ideas con el panel de pictogramas de TEVI que con el teclado virtual. Con el fin de confirmar esta apreciación, el equipo de investigación procesó estadísticamente los tiempos registrados y se obtuvieron novedosos resultados.

\section{Resultados y discusión}

Para determinar si existían diferencias estadísticamente significativas de tiempos de comunicación de los niños usando el teclado virtual y después el panel de pictogramas, se realizó un análisis de varianza, usando el programa SPSS (IBM Corp, 2012). En el análisis se aplicó el Modelo Lineal General Univariante, ya que en este estudio existe una única variable dependiente, que es el tiempo que tarda el alumno en comunicarse usando el teclado virtual en primera instancia y luego usando panel de pictogramas. En la figura 6 se muestra el diagrama de barras de los resultados registrados con los 35 niños del Centro Educativo Especial Ambato (CEEA).

Se puede observar que la media de tiempo en el teclado virtual es mayor a la media de tiempo registrado con el panel de pictogramas. Este efecto también ocurre con los cuarenta niños del Centro Educativo Especial Maximiliano Spiller (CEEMS). Cabe indicar que para el análisis estadístico de los tiempos de comunicación registrados, como factores de interés para los CEE, se consideraron la edad, el grado de limitación motriz, el tipo de entrada (Teclado Virtual, Panel de Pictogramas) y la combinación de estos factores.

Tiempos Promedio

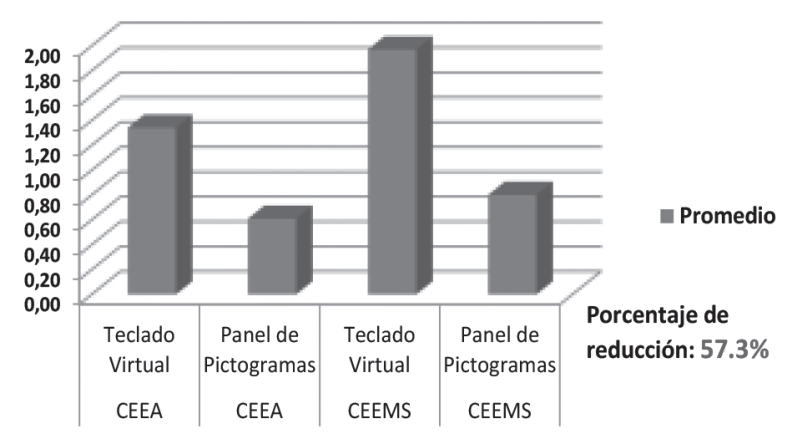

FIGURA 6

Tiempos promedios registrados con los CEE con el Teclado Virtual y el Panel de Pictogramas de TEVI. 
Estos resultados confirman que hubo una disminución del $57.3 \%$ al usar el panel de pictogramas. En el cuadro 4, se observa que el nivel de significación del modelo fue de $0,000 \mathrm{y}$ es menor que el valor del estadístico $\alpha$ de 0,05 .
Esto indica que existen diferencias estadísticamente significativas entre las varianzas de los tiempos de comunicación de los niños con dificultades en el lenguaje a causa de la discapacidad motriz.

CUADRO 4

Análisis Estadístico en SPSS

Prueba de los efectos inter-sujetos

\begin{tabular}{|c|c|c|c|c|c|}
\hline Origen & $\begin{array}{l}\text { Suma de } \\
\text { cuadrad } \\
\text { os tipo } \\
\text { III }\end{array}$ & Gl. & $\begin{array}{l}\text { Media } \\
\text { cuadrá } \\
\text { tica }\end{array}$ & $\mathbf{F}$ & Sig. \\
\hline Modelo corregido & $16,904^{0}$ & 7 & 2.415 & 9.122 & 0,000 \\
\hline Intersección & 31,902 & 1 & 31.902 & $\begin{array}{l}120.51 \\
2\end{array}$ & 0,000 \\
\hline Centro Educativo & 0,631 & 1 & 0,631 & 2.384 & 0,125 \\
\hline TipodeEntrada & 8,316 & 1 & 8,316 & 31,414 & 0,000 \\
\hline Limitacion & 0,001 & 2 & 0,000 & 0,001 & 0,999 \\
\hline $\begin{array}{l}\text { CentroEducativo*Tipod } \\
\text { eEntrada }\end{array}$ & 1,375 & 1 & 1,375 & 5,194 & 0,054 \\
\hline $\begin{array}{l}\text { CentroEducativo*Limita } \\
\text { cion }\end{array}$ & 0,000 & 0 & . & . & . \\
\hline $\begin{array}{l}\text { TipodeEntrada*Limitaci } \\
\text { on }\end{array}$ & 1,375 & 2 & 0.000 & 0,001 & 0,999 \\
\hline $\begin{array}{l}\text { CentroEducativo*Tipod } \\
\text { eEntrada*Limitacion }\end{array}$ & 0,000 & 0 & . & . & . \\
\hline Error & 32,825 & 124 & 0,265 & & \\
\hline Total & 123,142 & 132 & & & \\
\hline Total Corregido & 49,729 & 131 & & & \\
\hline
\end{tabular}

Además de los resultados cuantitativos, se reportan novedosos resultados cualitativos, después de realizar entrevistas no estructuradas y encuestas a los maestros, donde el $100 \%$ de ellos concuerdan que el TEVI puede ser muy útil para desarrollar habilidades de lenguaje y alfabetización. Indicaron que el sistema se podría utilizar con niños y adultos con algún otro tipo de discapacidad o con niños sin discapacidad en la etapa escolar inicial.

Los maestros mencionaron también que el audio asociado a cada pictograma resulta de suma utilidad, ya que promueve la verbalización de las palabras. Además, que la característica para cambiar los pictogramas facilita la adaptación del software a necesidades particulares de cada niño, e incluso podría ser utilizado por padres de familia en el hogar.
Las observaciones realizadas por el equipo de investigación permitieron descubrir que los niños con problemas de movilidad leves pudieron usar el mouse para señalar las teclas o pictogramas, siendo la interacción con los pictogramas más fluida que el teclado virtual.

Con base en estos resultados, se puede resumir que el uso de la tecnología de la información y comunicación favorece el desarrollo de destrezas en comunicación y ofrece un potencial en el proceso de aprendizaje en el aula (Jácome, 2013: 31). La flexibilidad y parametrización de TEVI también constituye una ventaja frente a herramientas similares. Esta característica facilita que maestros y especialistas puedan crear estrategias de aprendizaje personalizadas. Este tipo de tácticas pueden fortalecer también el desarrollo de otro tipo de habilidades, tales 
como la atención, la concentración, la memoria y la motivación.

\section{Conclusiones}

El trabajo colaborativo del equipo de investigación, en coordinación con autoridades, maestros, padres de familia y niños, fue fundamental para la realización de la presente investigación. Las primeras entrevistas y encuestas, realizadas a los directores y maestros de los CEE, dieron indicios de la importancia de crear una solución computacional que ayude a mejorar la calidad de vida de niños con discapacidad motriz y problemas de comunicación oral.

El acompañamiento de terapeutas y de especialistas permitió que se desarrollara TEVI como una solución tecnológica para apoyar la inclusión educativa de niños con discapacidad y, su vez, soporte la labor de maestros en el aula.

La herramienta tecnológica TEVI fue diseñada con una interfaz amigable y accesible, apoyada con elementos visuales y auditivos para fortalecer la estimulación sensorial perceptiva. Se tomó en cuenta la portabilidad y por eso se estructuró la información con un gestor con base de datos livianos. Para el procesamiento de la información se implementaron algoritmos de texto predictivo y procesamiento del lenguaje natural.

La evaluación de TEVI en un contexto real permitió identificar la utilidad en aspectos de comunicación y su potencial en aspectos de aprendizaje. Se evidenció una reducción de 57.3 $\%$ en el tiempo de comunicación del panel de pictogramas frente al teclado virtual.

Estos resultados parecen mostrar un notable aporte para niños con problemas del lenguaje. Sin embargo, no se puede concluir que mejoró significativamente la comunicación con su entorno y desarrollo de aprendizajes en el aula. Para poder determinar una contribución en estas áreas se requiere una investigación longitudinal, que queda abierta para un trabajo futuro.

\section{Trabajo futuro}

Esta experiencia ha permitido identificar otras necesidades latentes de comunicación y aprendizaje, tales como: la comunicación entre pares, la inclusión educativa y tecnológica, el desarrollo de destrezas en el tiempo. También, queda la interrogante de si la herramienta puede adaptarse a niños y niñas con algún otro tipo de discapacidad, como la intelectual. Esto último a raíz de que en los centros de educación especial donde se realizó esta investigación se identificaron niños y niñas con Síndrome de Down. Finalmente, sería muy interesante investigar sobre el desarrollo de estrategias de aprendizaje que puedan crear maestros y especialistas con la herramienta.

\section{Agradecimientos}

Un reconocimiento a la Universidad Tecnológica Indoamericana por el apoyo financiero otorgado al proyecto de investigación No.078.025.2012. Un agradecimiento especial a todos los niños y niñas de los Centros de Educación Especial Ambato y Maximiliano Spiller, así como a las autoridades y maestras por la apertura y participación en el desarrollo de esta investigación.

\section{Referencias}

Acurio Recalde, Monica Anabelly. 2013. Análisis de la atención a las personas con discapacidad a través de los programas y servicios de accesibilidad y recreación, para grupos de atención prioritaria en la Provincia de Cotopaxi en el año 2012. Tesis de Maestría. Universidad Técnica particular de Loja.

Administración del Ec. Rafael Correa Delgado Presidente Constitucional de la República del Ecuador. (31 de Marzo del 2011). Registro Oficial. [417]. Ley Orgánica de Educación Intercultural. [Segundo suplemento]. Recuperado de: http://planipolis. iiep.unesco.org/upload/Ecuador/Ecuador Ley_organica_educacion_intercultural. pdfConsulta: 16 de mayo del 2015. 
Agüero, Pablo. 2012. Síntesis de voz aplicada a la traducción voz a voz. Tesis Doctoral. Universidad Politécnica de Cataluña.

Asterisk. 2009. Text-To-Speech (TTS) y Automatic Speech Recognition (ASR). Disponible en: http://www.wikiasterisk.com/index.php?title=TTS_y_ASR. Consulta: 19 de junio del 2014.

Barreto, Armando et al. 2000. «A practical EMG-based human-computer interface for users with motor disabilities». En: Revista de Rehabilitation Research and Development. XXXVII (11): 53-64.

Beck, Kent. 2004. Extreme Programming Explained: Embracing Change. Segunda Edición. EEUU: Addison Wesley.

Bradski, Gary et al. 2005. "Learning-Based computer vision with intel's open source computer vision library». En: Revista Intel Technol IX (2): 119-130.

Brogna, Patricia. 2009. Visiones y revisiones de la Discapacidad. México: Fondo de la Cultura Económica.

CONADIS. 2013. Agenda Nacional para la Igualdad en Discapacidades 2013-2017. Disponible en: http://www.planificacion.gob.ec/wp-content/uploads/downloads/2014/09/Agenda-Nacional-paraDiscapacidades.pdf. Consulta: 23 de abril del 2014.

Cordero Monge, Sergio. 2009. «Algunas consideraciones sobre el lenguaje común y el lenguaje técnico». En: Revista Káñina, Revista de Artes y Letras de la Universidad de Costa Rica. XXXIII (Especial):75-80.

Cortez, Augusto et al. 2009. «Procesamiento de lenguaje natural». En: Revista de
Ingeniería de Sistemas e Informática. XI (2):45-54. Perú: Universidad Nacional Mayor de San Marcos.

Desmet, Pieter et al. 2012. «Development and application of a pictorial mood-reporting instrument». En: Conference de Proceedings of the 8th International Design and Emotion: 11-25. Central Saint Martin College of Art \& Design, London (UK).

Espejo Cárdenas, Silvia et al. 2009. Estudio sobre el uso del sistema de comunicación aumentativo y aumentativo y alternative SC@UT. España: Editorial de la Universidad de Granada.

Estévez, Roberto. 2013. Análisis estadístico, demográfico y Epidemiológico de las discapacidades en el Ecuador. Tesis maestría. Ecuador: Universidad Técnica Particular de Loja.

González, Gabriel et al. 2013. «Evaluación de técnicas para la introducción de texto en Entornos Virtuales Inmersivos». En: VIII Congreso de Interacción PersonaOrdenador, Interacción (5): 313-32. España.

IBM Corporation. 2012. IBM SPSS Statistics for Windows. Versión 21.0. Disponible en: http://www-01.ibm.com/support/docview. wss?uid=swg21476197. Consulta: 21 de mayo del 2014.

Instituto Nacional de Estadísticas y Censos (INEC). 2015. Tecnología de la Información y Comunicación (TIC'S) 2015. Disponible en: http://www.ecuadorencifras.gob.ec/documentos/webinec/Estadisticas Sociales/TIC/2015/ Presentacion_TIC_2015.pdf. Consulta: 12 de septiembre del 2015. 
Jácome, Ligia. 2013. «Desarrollo de las destrezas cognitivas Vinculadas a las Matemáticas en los centros de educación básica del Cantón Ambato y las Tecnologías de Información y Comunicación». En: Revista Cienciamérica I (2): 18-23.

Klever, Boris y Niels Birbaumer. 2005. «Direct Brian Communication: neuroelectric and metabolic approaches at Tubingen». En: Revista de Cognitiving processing VI (1): 65-74.

Kro'lak, Aleksandra y Pawel Strumiłło. 2015. «Real Time Drowsy Driver Identification Using Eye Blink Detection». En: Revista de Advanced Research in Computer Science and Software Engineering. V (9): 335-339.

Kro'lak, Aleksandra y Pawel Strumiłło. 2009. «Eye-blink controlled human-computer interface for the disabled». En: Revista Advances Intel Soft Compute XI (4): 409-419.

Kumaran, Raghunandan et al. 2007. «Myoelectric signals for multimodal speech recognition». En: Revista Proc Interspeech'25Eurospeech. LIV (4): 694-699.

Leoni de León, Jorge. 2011. «DIGALO: Herramienta de apoyo básico para estudiantes de L2**»». En: Revista Káñina, Rev. Artes y letras. Univ. Costa Rica. XXXV (2): 63-67.

Maggio, Mariana. 2004. «Intervención Centrada en la Familia. Programa Hanen para Padres de Niños con TEL». Ponencia en: II Jornada sobre TEL. Asociación Valenciana de Padres de Niños con TEL (AVATEL). Valencia-España.

Pérez de la Maza, Luis. 2002. Programa de Estructuración Ambiental Por Ordenador para personas con Trastornos del Espectro autista: PEAPO. Madrid, España: Centro PAUTA. Disponible en: http://diversidad. murciaeduca.es/tecnoneet/docs/2002/322002.pdf. Consulta: 24 de Junio del 2015.

Perrinet, Jonathan et al. 2009. "Adaptación de una aplicación de e-Learning a t-Learning». En: V Congreso Iberoamericano de Telemática: 38-44. España.

Pinheiro, Carlos et al. 2011. «Alternative communication systems for people with severe motor disabilities». En: Revista BioMedical Engineering. XVI (3): 10-31.

Prabhu, Vijit y Girijesh Prasad. 2009. «Designing a virtual keyboard with multi-modal access for people with disabilities». En: Revista IEEE. XXIV (2): 1133 - 1138.

Robles Bello, María y David Sánchez Teruel. 2014. Evaluación e intervención en atención infantil temprana: hallazgos recientes y casos prácticos. España: Universidad de Jaén.

Rodríguez, Wanda. 2002. «Herramientas culturales y transformaciones mentales: De los jeroglíficos al Internet». En: Revista de Ciencias de la conducta. XVII (1): 14-17.

Secretaría Nacional de Planificación y Desarrollo. 2013. Plan Nacional para el Buen Vivir 2013-2017. Disponible en: http://www. buenvivir.gob.ec/descarga-objetivo. Consulta: 27 de septiembre del 2014.

SQLite Copyright. 2014. SQLite. Disponible en: https://system.data.sqlite.org/index.html/ doc/trunk/www/index.wiki. Consulta: 12 de abril del 2013.

Vallez, Mari y Rafael Pedraza. 2007. «El Procesamiento del Lenguaje Natural en 
la Recuperación de Información Textual $y$ áreas afines». En: Hipertext.net. (5). Disponible en: https://www.upf.edu/hipertextnet/numero-5/pln.html. Consulta: 3 de noviembre del 2014.

Wolpaw, Jonathan et al. 2002. Brain computer interfaces for communication and control. En: Elsevier. (113): 767-791. Disponible en: http://www.cs.cmu.edu/ tanja/BCI/ BCIreview.pdf

Zhai, Shumin et al. 2000. «The Metropolis Keyboard-An Exploration of Quantitative Techniques for Virtual Keyboard Design». En: In Proceedings of the 13th annual ACM symposium on User interface software and technology: 119-128. ACM.

\section{(9) $\Theta \Theta \Theta$}

Este obra está bajo una licencia de Creative Commons Reconocimiento-NoComercial-SinObraDerivada 4.0 Internacional. 